\title{
Relationship between miR-204 and ANGPTL2 expression and diagnosis, pathological stage, and prognosis in patients with colon cancer
}

\author{
Chenliang Wang', Rongfei Tan ${ }^{2}$, Lizi Peng ${ }^{1}$, Jing Zhang ${ }^{1}$ \\ ${ }^{1}$ Department of Pathology, First People's Hospital of Jiujiang City, Jiujiang, China; ${ }^{2}$ Laboratory Department, Ruichang Maternal and Child Health \\ Hospital, Jiujiang, China \\ Contributions: (I) Conception and design: C Wang; (II) Administrative support: C Wang; (III) Provision of study materials or patients: L Peng; (IV) \\ Collection and assembly of data: R Tan; (V) Data analysis and interpretation: J Zhang; (VI) Manuscript writing: All authors; (VII) Final approval of \\ manuscript: All authors. \\ Correspondence to: Chenliang Wang. Department of Pathology, First People’s Hospital of Jiujiang City, Jiujiang, China. Email: jjsdyrmyytg@163.com.
}

Background: Angiopoietin-like protein 2 (ANGPTL2) is linked to various tumors. MicroRNA-204 (miR-204) is associated with colorectal cancer (CRC). Bioinformatic analysis has demonstrated a targeting relationship between miR-204 and ANGPTL2. The present study aimed to investigate the role of miR-204 in the proliferation and apoptosis of colorectal tumor cells.

Methods: Colorectal tumor tissues were collected. Normal colon mucosa was used as a control. The relationship between miR-204 and ANGPTL2 expression and tumor stage and prognosis was analyzed. The dual-luciferase reporter assay confirmed targeted regulation between miR-204 and ANGPTL2. SW480 cells were allocated to the miR-NC group and the miR-204 mimic group, followed by apoptotic analysis using flow cytometry and cellular proliferation analysis using EdU staining.

Results: Compared with normal colonic mucosa, miR-204 expression was decreased in colorectal tumor tissues and ANGPTL2 expression was increased, which correlated with TNM staging. The prognosis of patients with low miR-204 expression and high ANGPTL2 expression was worse than for patients with high miR-204 expression and low ANGPTL2 expression. The dual-luciferase reporter assay confirmed a targeting regulation relationship between miR-204 and ANGPTL2. Transfection of miR-204 mimic significantly inhibited the expression of ANGPTL2 and cell proliferation in SW480 cells and promoted apoptosis.

Conclusions: Downregulating miR-204 expression plays a vital role in upregulating ANGPTL2 expression and promoting the pathogenesis of CRC. MiR-204 is able to hinder the proliferation of colorectal tumor cells and encourage apoptosis by targeting the inhibition of ANGPTL2 expression.

Keywords: Colorectal cancer (CRC); microRNA-204 (miR-204); angiopoietin-like protein 2 (ANGPTL2); proliferation; apoptosis

Submitted Jun 04, 2021. Accepted for publication Aug 12, 2021.

doi: $10.21037 /$ tcr-21-1385

View this article at: https://dx.doi.org/10.21037/tcr-21-1385

\section{Introduction}

Colorectal cancer (CRC) is a malignant tumor of the digestive tract and the third most commonly diagnosed cancer (1). The characteristics of CRC include high incidence, occultation, rapid disease progression, and poor prognosis (2-4). It has been reported that key driver gene mutations, such as BRAF, KRAS, NRAS, HER2 and etc., play an important role in the progression and metastasis of CRC. Certainly, functions of more non-classical genes in CRC are still needed to explore.

Angiopoietin-like protein 2 (ANGPTL2) is an important regulator of chronic inflammation and metabolic 
abnormalities in obesity; however, research findings also link abnormal expression of ANGPTL2 to multiple tumors $(5,6)$. Studies have shown that abnormally elevated expression of $A N G P T L 2$ plays a vital role in promoting the development, progression, and drug resistance of CRC (7-9).

MiR-204 is an important epigenetic regulatory molecule. The expression of miR-204 in the tumor tissues and cells of colorectal patients has been shown to be considerably downregulated (10-12), implying that miR-204 may be involved in colorectal tumorigenesis by playing a tumorsuppressor role. Bioinformatic analysis has demonstrated that there is a targeted complementary binding site between miR-204 and ANGPTL2 mRNA, suggesting a possible regulatory effect between the two. However, there are studies focused on the functions of only miR-204 or only $A N G P T L 2$ in colorectal cancer. The relationship between miR-204 and ANGPTL2 expression and diagnosis, pathological stage, and prognosis in patients with colon cancer is still absent. Therefore, this study examined the expression profiles of miR-204 and ANGPTL2 in the tumor tissues of CRCs, analyzed the correlation between the level of miR-204 and ANGPTL2 expressions and tumor stage and prognosis, and investigated whether miR-204 can influence the regulation of $A N G P T L 2$ expression and affect CRC cell proliferation and apoptosis. We present the following article in accordance with the STARD reporting checklist (available at https://dx.doi.org/10.21037/tcr-21-1385).

\section{Methods}

\section{Main reagents and materials}

CRC SW480 cells were purchased from KeyGen Biotech Co., Ltd. (Nanjing, China); Dulbecco's Modified Eagle Medium (DMEM) was purchased from Biological Industries (BeitHaemek, Israel); fetal bovine serum (FBS) was purchased from SeraPro (Germany); quantitative reverse transcription polymerase chain reaction ( $\mathrm{q}$ RT-PCR) SuperMix was purchased from TransGen Biotech Co., Ltd. (Beijing, China); TRIzol and Lipofectamine (Lipo) 2000 were purchased from Invitrogen (Waltham, MA, USA); miR$\mathrm{NC}$ and miR-204 mimic were purchased from RiboBio Co., Ltd. (Guangzhou, China); EdU cell proliferation flow assay kit was purchased from Sigma-Aldrich (St Louis, MO, USA); rabbit anti-ANGPTL2 antibody was obtained from Abacm (Cambridge, MA, USA); rabbit anti- $\beta$-actin antibody was purchased from Santa Cruz Biotechnology Inc. (Dallas,
TX, USA); HRP-conjugated secondary antibody was purchased from Sangon Biotechnology (Shanghai, China); pMIR plasmid was purchased from BioVector NTCC Inc. (Beijing, China); Dual-Glo Luciferase Assay System was purchased from Promega Corporation (Madison, WI, USA); radio-immune precipitation assay (RIPA) lysate was purchased from Hangzhou Multi Science (Lianke) Biotech Co. (Hangzhou, China); BeyoECL Plus chemiluminescence solution and Annexin V-FITC Apoptosis Detection Kit with propidium iodide (PI) were purchased from Suo Labao Bio (Beijing, China).

\section{Clinical data}

Eighty patients with CRC who were treated in our hospital from September 2016 to July 2018 were enrolled in the study. Tumor tissue specimens removed during patient operations were collected and confirmed by histopathological examination. Among them, there were 20 cases in TNM stage I, 18 cases in stage II, 23 cases in stage III, and 29 cases in stage IV. Specimens of normal intestinal mucosa, collected from 80 colonoscopies performed in our hospital, were used as controls. All procedures performed in this study involving human participants were in accordance with the Declaration of Helsinki (as revised in 2013). The study was approved by ethics committee board of First People's Hospital of Jiujiang City and informed consent was taken from all the patients.

\section{Dual luciferase reporter gene assay}

A full-length 3'-UTR fragment of the ANGPTL2 gene or that containing the mutant was amplified using the HEK293T cell genome as a template. It was then cloned into the pMIR vector, transformed into DH5 $\alpha$ competent cells, and the correct plasmid was sequenced and named as pMIR-ANGPTL2-WT, pMIR-ANGPTL2-MUT. pMIRANGPTL2-WT (or pMIR-ANGPTL2-MUT) and miR-204 mimic (or miR-NC) were cotransfected into HEK293 T cells with Lipo 2000 reagent. After 48 hours of incubation, relative fluorescence intensity was determined using the Dual-Glo Luciferase Assay System.

\section{Cell transfection and grouping}

CRC SW480 cells were then cultured in a DMEM medium which contained $10 \%$ FBS and $1 \%$ streptomycin at $37{ }^{\circ} \mathrm{C}$ with $5 \% \mathrm{CO}_{2}$. Next, $0.125 \%$ trypsin was used to digest cells, 
which were then subcultured at a ratio of $1: 4$ to $1: 5$. Cells in the logarithmic growth phase were assigned to the miR-NC transfection group and miR-204 mimic transfection group. The general procedure for transfection was as follows: $10 \mu \mathrm{L}$ of Lipo 2000, $50 \mathrm{nmoL}$ miR-NC, and $50 \mathrm{nmoL}$ miR141 mimic were diluted with $100 \mu \mathrm{L}$ of serum-free OptiMEM, respectively, and incubated for 5 minutes at room temperature. Lipo 2000 and miR-NC, and Lipo 2000 and miR-141 mimic, respectively, were mixed and incubated for 20 minutes at room temperature. The transfectants were then added to the cell culture medium, mixed and cultured for 72 hours.

\section{Flow cytometry detection of cell proliferation}

The SW480 cells from the two transfection groups were collected by trypsinization and resuspended in DMEM complete medium containing $10 \%$ FBS. After incubating with $10 \mu \mathrm{M}$ of $\mathrm{EdU}$ at $37^{\circ} \mathrm{C}$ for 2 hours, the cells were inoculated on the culture plate for 48 hours and then collected after trypsin digestion. Paraformaldehyde was added to fix the cells, after which the cells were washed once in phosphate buffer saline (PBS) and then $100 \mu \mathrm{L}$ permeabilization solution and $500 \mu \mathrm{L}$ reaction test solution was added. After incubation at room temperature for 30 minutes in the dark, the cells were centrifuged and resuspended in $500 \mu \mathrm{L}$ wash buffer and cell proliferation was measured by FC500 MCL flow cytometry.

\section{Flow cytometry detection of apoptosis}

After transfection, SW480 cells were resuspended in an Annexin V Binding Buffer of $100 \mu \mathrm{L}$ and then mixed with $5 \mu \mathrm{L}$ of Annexin V-FITC and $10 \mu \mathrm{L}$ of PI. After 15 minutes of incubation at room temperature, $400 \mu \mathrm{L}$ of Annexin V Binding Buffer was added and cell apoptotic activity was examined using the FC500 MCL flow cytometer.

\section{qRT-PCR detection of gene expression}

The relative expression of genes was detected by one-step qRT-PCR using TransScript Green One-Step qRT-PCR SuperMix in a $20 \mu \mathrm{L}$ reaction mixture including: $1 \mu \mathrm{g}$ of Template RNA, $0.2 \mu \mathrm{M}$ of pre-primer, $0.2 \mu \mathrm{M}$ of postprimer, $10 \mu \mathrm{L}$ of $2 \times$ TransStart Tip Green qPCR SuperMix, $0.4 \mu \mathrm{L}$ of One-Step RT Enzyme Mix, $0.4 \mu \mathrm{L}$ of Passive Reference Dye II, and RNase-free water. The settings for the qRT-PCR reaction were as follows: $45^{\circ} \mathrm{C}, 5$ minutes;
$94{ }^{\circ} \mathrm{C}, 30$ seconds; $94{ }^{\circ} \mathrm{C}, 5$ seconds; $60{ }^{\circ} \mathrm{C}, 30$ seconds $\times 40$ cycles. Gene expression was then detected on a Bio-Rad CFX96 real-time PCR instrument.

\section{Western blot}

RIPA Lysis Buffer was used to extract the protein and the concentration was determined. Sodium dodecyl sulphatepolyacrylamide gel electrophoresis (SDS-PAGE) was used to separate $40 \mu \mathrm{g}$ of protein, which was then transferred onto polyvinylidene difluoride (PVDF) membrane, blocked with $5 \%$ skim milk powder in phosphate buffered saline with Tween (PBST) for 60 minutes at room temperature, and incubated at $4{ }^{\circ} \mathrm{C}$ overnight with the primary antibodies (ANGPTL2, $\beta$-actin; dilution ratios: 1:1,000, 1:5,000, respectively). After being washed 3 times with PBST, the membrane was incubated at room temperature with HRPconjugated secondary antibody (1:5,000, 60 minutes) and then washed 3 times with PBST. Equal parts of BeyoECL Plus Solution A and B were mixed and then added to the Western blot membrane and incubated for 2-3 minutes, followed by exposure and development of the film.

\section{Statistical analysis}

Statistical analysis was carried out using SPSS 18.0 software. The measurement data are expressed in the form of mean \pm standard deviation (SD). T-test was used to compare the results between the two groups. Comparison between multiple groups was by one-way ANOVA and then by the Bonferroni method. The Mann-Whitney $U$ test compared miR-204 and ANGPTL2 mRNA expressions in normal intestinal mucosa and intestinal cancer tissues. The CRC patients were allocated to separate groups based on the median expression of miR-204 and ANGPTL2 mRNA. The log-rank test was used to analyze the relationship between miR-204 and ANGPTL2 mRNA expression and survival and prognosis. $\mathrm{P}<0.05$ was considered statistically significant.

\section{Results}

\section{Abnormal expression of miR-204 and ANGPTL2 in CRC}

The results indicated that the expression of miR-204 was significantly decreased in the tumor tissues of CRC patients compared with normal intestinal mucosa, and the expression of miR-204 was significantly lower in TNM stages III-IV intestinal cancer tissue than in TNM 
A

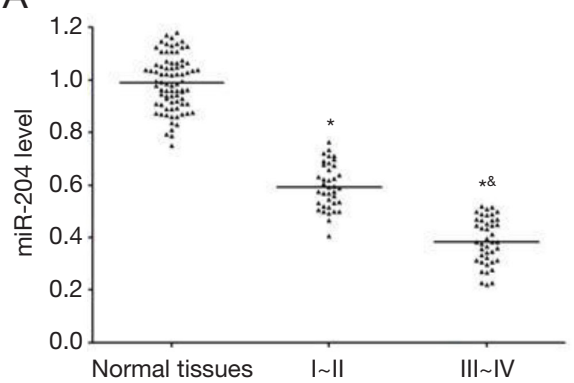

B

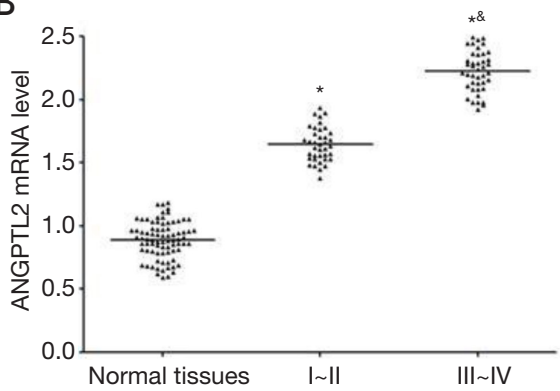

Figure 1 Abnormal expression of miR-204 and ANGPTL2 in colorectal cancer. (A) qRT-PCR detection of miR-204 expression in intestinal cancer tissues; (B) qRT-PCR detection of ANGPTL2 mRNA expression in intestinal cancer tissues. *, $\mathrm{P}<0.05$ compared with normal intestinal mucosa; ${ }^{\&}, \mathrm{P}<0.05$ compared with stages $\mathrm{I}-\mathrm{II}$ intestinal cancer tissue.
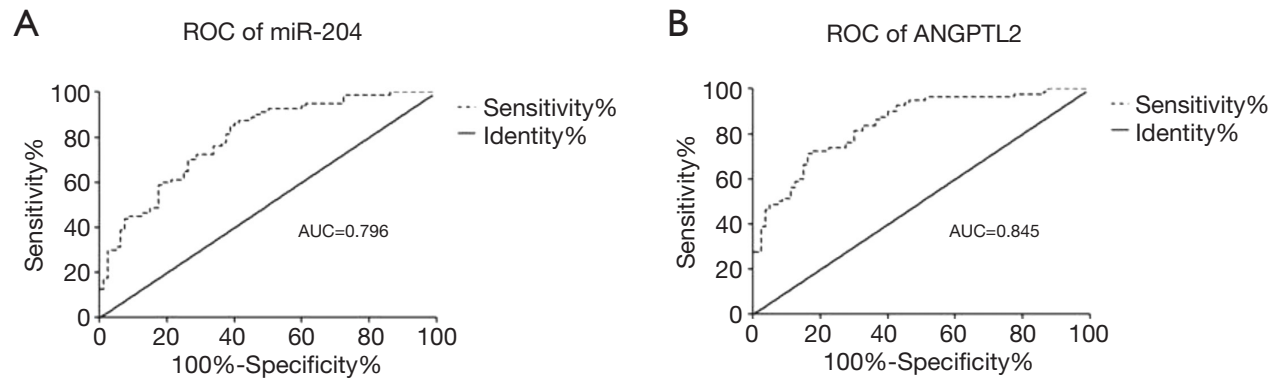

Figure 2 ROC curve analysis of miR-204 and ANGPTL2 in the diagnosis of colorectal cancer. (A) ROC curve analysis of the diagnostic value of miR-204 for colorectal cancer; (B) ROC curve analysis of the diagnostic value of ANGPTL2 for colorectal cancer.

stages I-II intestinal cancer tissue (Figure $1 A$ ). The results highlighted that $A N G P T L 2$ mRNA expression was significantly higher in the tissues of CRC patients compared to normal intestinal mucosa tissue, and the expression of ANGPTL2 mRNA in TNM stages III-IV cancer tissue was significantly higher than that in TNM stages I-II intestinal cancer tissue (Figure 1B).

\section{Diagnostic value of miR-204 and ANGPTL2 in CRC}

The results of receiver operating characteristic (ROC) curve analysis showed that miR-204 (Figure $2 A$ ) and $A N G P T L 2$ mRNA (Figure $2 B$ ) expression had a high diagnostic value for CRC, with the area under the curve (AUC) 0.796 and 0.845 , respectively.

\section{Association between poorer prognosis and decreased expression of miR-204 along with increased expression of ANGPTL2}

Based on the median expression of miR-204 and ANGPTL2
mRNA, 80 patients with CRC were divided into a high expression group and low expression group. The results showed that patients with lower expression of miR-204 had worse prognosis compared with patients with higher expression of miR-204 (log-rank test $\chi^{2}=3.955, \mathrm{P}=0.047$ ) (Figure $3 A$ ). In addition, patients with higher $A N G P T L 2$ mRNA expression had worse prognosis compared to patients with lower ANGPTL2 mRNA expression (log-rank test $\chi^{2}=4.428, \mathrm{P}=0.035$ ) (Figure $3 B$ ).

\section{Targeted regulation relationship between miR-204 and ANGPTL2}

TargetScan's online prediction tool showed that there was a targeted complementary binding site between miR-204 and the 3'-UTR of ANGPTL2 mRNA (Figure 4A). The dual luciferase reporter assay indicated that the transfection of miR-204 mimic significantly reduced the relative luciferase activity of pMIR-ANGPTL2-WT transfected HEK293T cells. However, miR-NC transfection did not affect the relative luciferase activity in HEK293T cells transfected 

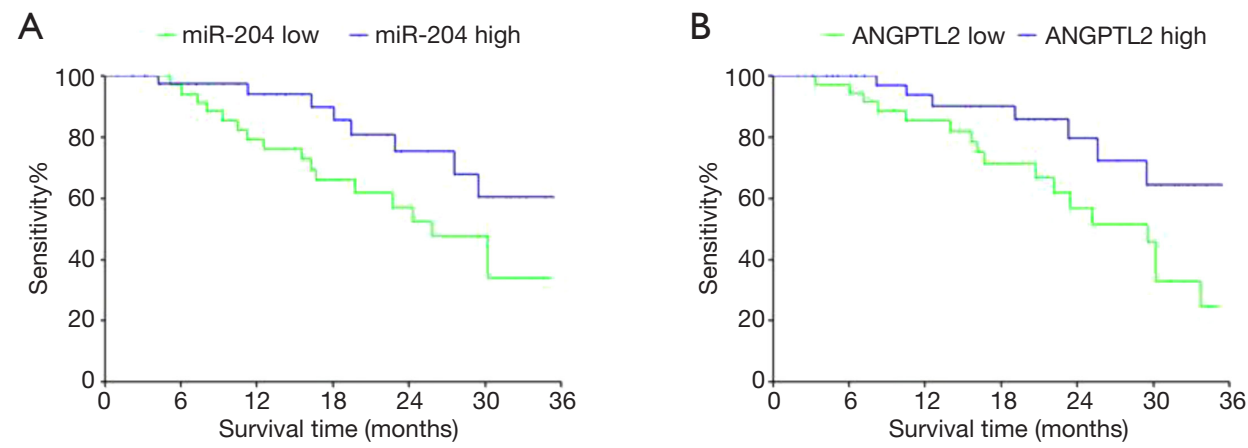

Figure 3 Survival curve analysis of the relationship between miR-204, ANGPTL2 and prognosis of colorectal tumors. (A) Survival curve analysis of the relationship between miR-204 and the prognosis of colorectal tumors; (B) survival curve analysis of the relationship between ANGPTL2 and the prognosis of colorectal cancer.

A

$$
\begin{aligned}
& \text { 5' GUUUUGUGUAUAUAAAGGGGAG 3' pMIR-ANGPTL2-WT } \\
& \text { 3' UCCGUAUCCUACUGUUUCCCCUU 5' hsa-miR-204 } \\
& \text { 5' GUUUUGUGUaUAUACAGAGUCG 3' pMIR-ANGPTL2-MUT }
\end{aligned}
$$

B

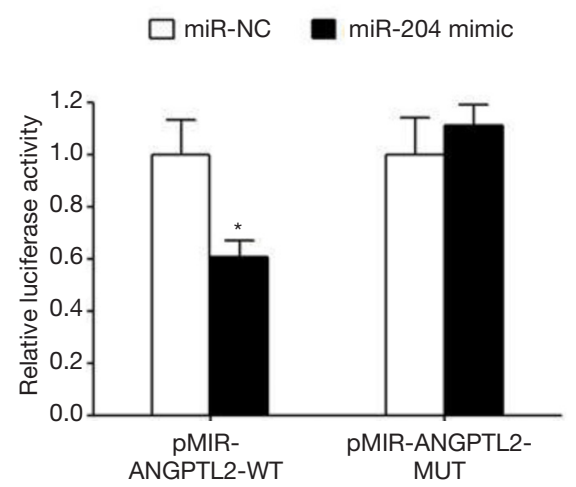

Figure $4 \mathrm{MiR}-204$ has a targeted regulatory relationship with ANGPTL2 mRNA. (A) A binding site between miR-204 and the 3'-UTR of ANGPTL2 mRNA; (B) results of the dual luciferase reporter assay. *, $\mathrm{P}<0.05$ compared to the miR-NC group.

with pMIR-ANGPTL2-WT. Transfection of miR204 mimic or miR-NC had little impact on the relative luciferase activity in pKIR-ANGPTL2-MUT transfected HEK293T cells (Figure 4B), indicating the presence of a targeted regulatory relationship between miR-204 and ANGPTL2 mRNA.

\section{Overexpression of miR-204 inbibits ANGPTL2 expression and proliferation of intestinal cancer cells and promotes apoptosis}

The qRT-PCR assay showed that miR-204 mimic transfection significantly downregulated the expression of ANGPTL2 mRNA in SW480 cells compared to the miR-NC group (Figure $5 A$ ). Results of the Western blot analysis revealed that the $A N G P T L 2$ protein expression in SW480 cells of the miR-204 mimic transfection group was considerably lower than that of the miR-NC group (Figure 5B). Flow cytometry results showed that transfection of miR-204 mimic significantly increased the apoptosis of SW480 cells (Figure 5C) while cell proliferation was significantly inhibited (Figure 5D).

\section{Discussion}

The ANGPTL2 gene is located in human chromosome $9 \mathrm{q} 34$ and encodes a protein consisting of 493 amino acids. ANGPTL2 is a secreted glycoprotein with a molecular mass of $57 \mathrm{kDa}$ and belongs to the angiopoietin protein family. It was first confirmed and cloned by Kim et al. in 1999. There is a relationship between the abnormal expression of ANGPTL2 and the occurrence, progression, invasion, metastasis, and prognosis of various malignancies, including lung cancer (5), breast cancer (6), liver cancer (13), and thyroid cancer (14). Studies have shown that an abnormally elevated expression of $A N G P T L 2$ plays a vital role in promoting carcinogenesis and drug resistance of colorectal malignancies (7-9). It has also been reported that $A N G P T L 2$ 
A

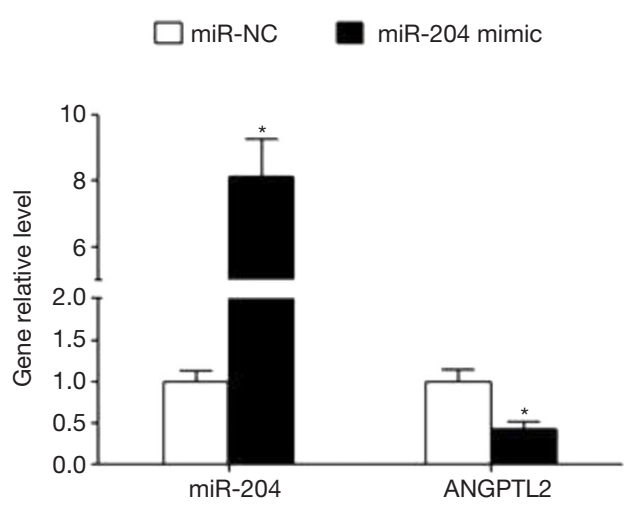

B

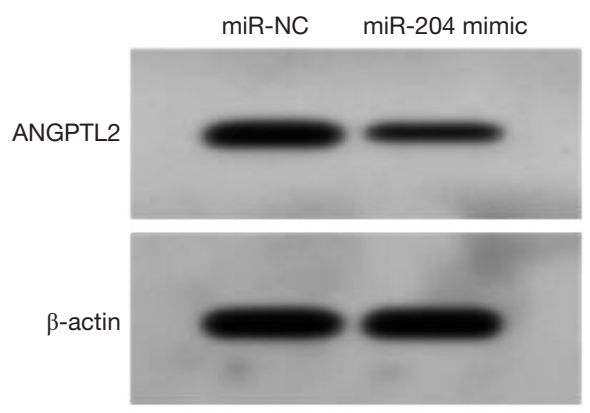

C

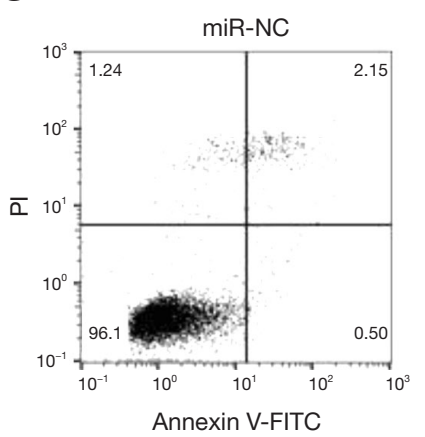

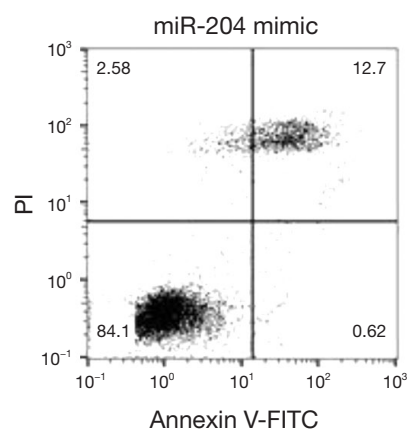
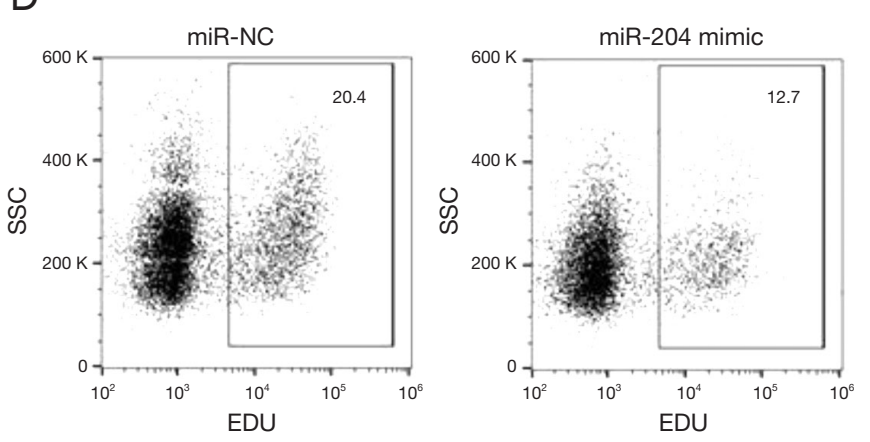

Figure 5 Overexpression of miR-204 hinders the expression of ANGPTL2 and proliferation of intestinal cancer cells and promotes apoptosis. (A) qRT-PCR detection of miR-204 and ANGPTL2 mRNA expression; (B) Western blot analysis of ANGPTL2 protein expression; (C) apoptosis flow detection; (D) cell proliferation flow detection. *, $\mathrm{P}<0.05$ compared to the miR-NC group.

promotes cancer progression via PI3K-AKT pathway and ERK/MAPK pathway $(15,16)$. In our study, we have demonstrated that ANGPTL2 promotes the proliferation of colorectal cancer cells, and meanwhile, PI3K-AKT pathway and ERK/MAPK pathway are typical proliferation-related pathways. Therefore, we speculate $A N G P T L 2$ promotes the proliferation of colorectal cancer via PI3K-AKT pathway and ERK/MAPK pathway, and we will prove the conclusion in the future.

MicroRNA (miRNA) is a non-coding, endogenous, small RNA molecule of eukaryotes that can bind complementarily to the 3'-untranslated region (3'-UTR) of the target mRNA in order to degrade mRNA or inhibit mRNA translation, thus regulating the expression of target genes and contributing to regulating biological processes such as cell proliferation, apoptosis, and migration. MiRNA expression and dysfunction are linked to several cancers, including lung carcinoma, ovarian carcinoma, bladder carcinoma, and breast carcinoma (17-20). The abnormal expression of miR-204 is correlated with the formation, progression, metastasis, and drug resistance of various tumors, such as liver cancer (21), gastric cancer (22), and non-small cell lung cancer (23). Studies have found that the expression of miR204 in the tumor tissues and cells of colorectal carcinoma patients was significantly reduced, suggesting that miR204 could potentially have a role as a tumor suppressor in colorectal tumors. MiR-204 can inhibit the growth and motility of colorectal cancer cells by downregulation of CXCL8. MiR-204-5p can inhibit proliferation and invasion and enhance chemotherapeutic sensitivity of colorectal cancer cells by downregulating RAB22A (10-12). Certainly, other molecular mechanisms of miR-204 in colorectal cancer still needs to explore.

The results of the present paper showed that compared with the normal intestinal mucosa of the control group, the expression of miR-204 in colorectal tumor tissue was 
substantially reduced while the expression of $A N G P T L 2$ was significantly increased. Further, the higher the TNM stage, the lower the miR-204 expression level and the higher the expression level of $A N G P T L 2$. ROC curve analysis showed that the detection of miR-204 and ANGPTL2 expression had high diagnostic value for CRC. Survival curve analysis suggested that decreased miR-204 expression and increased ANGPTL2 expression were predisposing factors for poor prognosis for colorectal tumors. Coincidentally, the present study also highlights that decreased expression of miR204 might upregulate $A N G P T L 2$ expression and play an indispensable role in colorectal carcinogenesis. The results of the dual luciferase gene reporter assay showed that there was targeted regulation between miR-204 and $A N G P T L 2$, which further confirmed that the expression of ANGPTL2 in colorectal tumors was associated with decreased expression of miR-204. In a study focusing on the relationship between miR-204 and CRC, Yin et al. (12) showed that the expression of miR-204 was considerably downregulated in tumor tissues of patients with CRC and that decreased expression of miR-204 was associated with a poor prognosis. The results of Shuai et al. (10) showed that the expression of miR-204 in colorectal cancer tissues and CRC cell lines was significantly decreased compared with the control. The results from the research conducted by $\mathrm{Wu}$ et al. (11) reinforced that miR-204 expression was decreased in an atypical manner in colorectal tumor cells while the expression of target gene HMGA2 was significantly increased. In this study, a notable decrease in the expression of miR-204 in colorectal tumor tissues was evident, suggesting that the decreased expression of miR-204 plays a role in CRC and is associated with poor prognosis, which aligns with the results of research by Yin et al. (12) and Shuai et al. (10).

Further, this study transfected miR-204 mimic to overexpress miR-204 in CRC SW480 cells and showed that an elevated expression of miR-204 can promote cell apoptosis and inhibit cell proliferation by targeting $A N G P T L 2$ expression. In a research paper that investigated the relationship between miR-204 and the biological effects of CRC cells, Yin et al. (12) showed that overexpression of miR-204 in CRC cells significantly inhibited the proliferation of CRC cells and reduced the growth ability and tumorigenicity of cancer cells in animals, hindered the migration and invasion of intestinal cancer cells, and enhanced the sensitivity of cells to chemotherapeutic drugs. The inhibitory effect of miR-204 on CRC is achieved by targeting inhibition of $R A B 22 A$ gene expression. Shuai et al. (10) showed that overexpression of miR-204 in CRC Caco-2 and HT-29 cells significantly inhibited cell proliferation and epithelial-mesenchymal transition (EMT) and attenuated cell migration and invasion through targeted inhibition of CXCL8. Li et al. (24) showed that the longchain noncoding RNA LncBRM can significantly inhibit the expression and impede the function of miR-204, thereby upregulating the expression and function of miR-204 target gene TPT1, encouraging and enhancing the proliferation, migration, and invasive capabilities of colorectal tumor cells. Wu et al. (25) showed that the expression of miR-204 was abnormally decreased in CRC cells and that decreased expression of miR-204 was associated with the increased expression of long-chain noncoding RNA PCAT6, which can inhibit miR-204 expression, increase the activity of the HMGA2/PI3K signaling pathway, and enhance the drug sensitivity of CRC cells to 5-fluorouracil (5-FU). In contrast, knockdown of PCAT6 expression increased the level of expression for miR-204 and reduced the resistance of CRC cells to 5-FU. The results of Bian et al. (26) showed that the expression and function of miR-204 in colorectal tumor cells were significantly inhibited by longchain noncoding RNA UCA1, which inhibits miR-204 and upregulates the expression and function of its target gene, CREB1, thereby promoting the proliferation of CRC cells, reducing apoptosis, and enhancing drug resistance of cells to 5-FU. The results of Wu et al. (11) demonstrated that overexpression of miR-204 in HCT-116 and SW480 cells can significantly inhibit the expression of HMGA2, attenuate cell proliferation, promote cell apoptosis, and reduce drug resistance to 5 -FU. These studies indicate that miR-204 attenuates the malignant biological characteristics of CRC cells, similarly to the results observed in the present study. This study links the targeting relationship between miR-204 and ANGPTL2, revealing that miR-204 has a vital role in targeting $A N G P T L 2$ expression and influencing proliferation and apoptosis of CRC cells. However, it remains unclear whether miR-204 could modulate the expression of $A N G P T L 2$ and exert an impact on the biological effects of colorectal tumor cells in vivo. Further research involving animal studies is needed.

\section{Conclusions}

The decreased expression of miR-204 plays a vital role in upregulating $A N G P T L 2$ expression and promoting the pathogenesis of CRC. MiR-204 can impede the proliferation of CRC cells and encourage apoptosis by 
targeting the inhibition of $A N G P T L 2$ expression.

\section{Acknowledgments}

Funding: None.

\section{Footnote}

Reporting Checklist: The authors have completed the STARD reporting checklist. Available at https://dx.doi. org/10.21037/tcr-21-1385

Data Sharing Statement: Available at https://dx.doi. org/10.21037/tcr-21-1385

Conflicts of Interest: All authors have completed the ICMJE uniform disclosure form (available at https://dx.doi. org/10.21037/tcr-21-1385). The authors have no conflicts of interest to declare.

Ethical Statement: The authors are accountable for all aspects of the work in ensuring that questions related to the accuracy or integrity of any part of the work are appropriately investigated and resolved. All procedures performed in this study involving human participants were in accordance with the Declaration of Helsinki (as revised in 2013). The study was approved by ethics committee board of First People's Hospital of Jiujiang City and informed consent was taken from all the patients.

Open Access Statement: This is an Open Access article distributed in accordance with the Creative Commons Attribution-NonCommercial-NoDerivs 4.0 International License (CC BY-NC-ND 4.0), which permits the noncommercial replication and distribution of the article with the strict proviso that no changes or edits are made and the original work is properly cited (including links to both the formal publication through the relevant DOI and the license). See: https://creativecommons.org/licenses/by-nc-nd/4.0/.

\section{References}

1. Clancy C, Khan S, Glynn CL, et al. Screening of exosomal microRNAs from colorectal cancer cells. Cancer Biomark 2016;17:427-35.

2. Zou SL, Chen YL, Ge ZZ, et al. Downregulation of serum exosomal miR-150-5p is associated with poor prognosis in patients with colorectal cancer. Cancer Biomark
2019;26:69-77.

3. Dimitriou N, Arandjelović O, Harrison DJ, et al. A principled machine learning framework improves accuracy of stage II colorectal cancer prognosis. NPJ Digit Med 2018;1:52.

4. Sun HY, Qu ZC, Liu DM, et al. Decreased expression of miR-551b predicts poor prognosis and promotes tumorigenesis by targeting PTP4A3 in human colorectal cancer. Eur Rev Med Pharmacol Sci 2019;23:5741-51.

5. Liu X, Yu X, Xie J, et al. ANGPTL2/LILRB2 signaling promotes the propagation of lung cancer cells. Oncotarget 2015;6:21004-15.

6. Masuda T, Endo M, Yamamoto Y, et al. Corrigendum: ANGPTL2 increases bone metastasis of breast cancer cells through enhancing CXCR4 signaling. Sci Rep 2015;5:9789.

7. Yoshinaga T, Shigemitsu T, Nishimata H, et al. Angiopoietin-like protein 2 as a potential biomarker for colorectal cancer. Mol Clin Oncol 2015;3:1080-4.

8. Toiyama Y, Tanaka K, Kitajima T, et al. Elevated serum angiopoietin-like protein 2 correlates with the metastatic properties of colorectal cancer: a serum biomarker for early diagnosis and recurrence. Clin Cancer Res 2014;20:6175-86.

9. Horiguchi H, Endo M, Miyamoto Y, et al. Angiopoietinlike protein 2 renders colorectal cancer cells resistant to chemotherapy by activating spleen tyrosine kinasephosphoinositide 3-kinase-dependent anti-apoptotic signaling. Cancer Sci 2014;105:1550-9.

10. Shuai F, Wang B, Dong S. MicroRNA-204 Inhibits the Growth and Motility of Colorectal Cancer Cells by Downregulation of CXCL8. Oncol Res 2018;26:1295-305.

11. Wu H, Liang Y, Shen L, et al. MicroRNA-204 modulates colorectal cancer cell sensitivity in response to 5 -fluorouracil-based treatment by targeting high mobility group protein A2. Biol Open 2016;5:563-70.

12. Yin $Y$, Zhang B, Wang W, et al. miR-204-5p inhibits proliferation and invasion and enhances chemotherapeutic sensitivity of colorectal cancer cells by downregulating RAB22A. Clin Cancer Res 2014;20:6187-99.

13. Gao L, Ge C, Fang T, et al. ANGPTL2 promotes tumor metastasis in hepatocellular carcinoma. J Gastroenterol Hepatol 2015;30:396-404.

14. Yang L, Sun R, Wang Y, et al. Expression of ANGPTL2 and its impact on papillary thyroid cancer. Cancer Cell Int 2019;19:204.

15. Yang L, Shu T, Liang Y, et al. GDC-0152 attenuates the malignant progression of osteosarcoma promoted by 
ANGPTL2 via PI3K/AKT but not $\mathrm{p} 38 \mathrm{MAPK}$ signaling pathway. Int J Oncol 2015;46:1651-8.

16. Yang LK, Zhu J, Chen YH, et al. Knockdown of Angiopoietin-Like Protein 2 Inhibits Proliferation and Invasion in Glioma Cells via Suppressing the ERK/MAPK Signaling Pathway. Oncol Res 2017;25:1349-55.

17. Lv X, Li CY, Han P, et al. MicroRNA-520a-3p inhibits cell growth and metastasis of non-small cell lung cancer through PI3K/AKT/mTOR signaling pathway. Eur Rev Med Pharmacol Sci 2018;22:2321-7.

18. Zhao R, Liu Q, Lou C. MicroRNA-299-3p regulates proliferation, migration and invasion of human ovarian cancer cells by modulating the expression of OCT4. Arch Biochem Biophys 2018;651:21-7.

19. Pardini B, Cordero F, Naccarati A, et al. microRNA profiles in urine by next-generation sequencing can stratify bladder cancer subtypes. Oncotarget 2018;9:20658-69.

20. Liu J, Yang L, Guo X, et al. Sevoflurane suppresses proliferation by upregulating microRNA-203 in breast cancer cells. Mol Med Rep 2018;18:455-60.

21. Yu Y, Wang Y, Xiao X, et al. MiR-204 inhibits hepatocellular cancer drug resistance and metastasis

Cite this article as: Wang C, Tan R, Peng L, Zhang J. Relationship between miR-204 and ANGPTL2 expression and diagnosis, pathological stage, and prognosis in patients with colon cancer. Transl Cancer Res 2021;10(8):3788-3796. doi: $10.21037 /$ tcr-21-1385 through targeting NUAK1. Biochem Cell Biol 2019;97:563-70.

22. Yuan H, Zhang J, Li F, et al. Sinomenine exerts antitumour effect in gastric cancer cells via enhancement of miR-204 expression. Basic Clin Pharmacol Toxicol 2019;125:450-9.

23. Huang G, Lou T, Pan J, et al. MiR-204 reduces cisplatin resistance in non-small cell lung cancer through suppression of the caveolin-1/AKT/Bad pathway. Aging (Albany NY) 2019;11:2138-50.

24. Li R, Zhu H, Yang D, et al. Long noncoding RNA lncBRM promotes proliferation and invasion of colorectal cancer by sponging miR-204-3p and upregulating TPT1. Biochem Biophys Res Commun 2019;508:1259-63.

25. Wu H, Zou Q, He H, et al. Long non-coding RNA PCAT6 targets miR-204 to modulate the chemoresistance of colorectal cancer cells to 5 -fluorouracil-based treatment through HMGA2 signaling. Cancer Med 2019;8:2484-95.

26. Bian $Z$, Jin L, Zhang J, et al. LncRNA-UCA1 enhances cell proliferation and 5-fluorouracil resistance in colorectal cancer by inhibiting miR-204-5p. Sci Rep 2016;6:23892.

(English Language Editor: A. Muijlwijk) 